\title{
Aportaciones de la paleohistología humana al estudio de biomateriales
}

\author{
NACARINO MENESES, C. ${ }^{1}$, CAMBRA-MOO, O. ${ }^{1}$, RODRÍGUEZ BARBERO, M.A., ${ }^{2}$ GONZÁLEZ MARTÍN, A. ${ }^{1}$ \\ 'Laboratorio de Poblaciones del Pasado (LAPP). Departamento de Biología. Facultad de Ciencias. Universidad Autónoma de Madrid. 28049 \\ Madrid, España - Spain. \\ ${ }^{2}$ Instituto de Cerámica y Vidrio. Consejo Superior de Investigaciones Científicas (CSIC). 28049. Madrid. España - Spain.
}

\begin{abstract}
El estudio en profundidad del hueso humano arqueológico puede aportar información relevante a la ciencia de los biomateriales, al informar sobre la evolución del proceso de implante de biovidrios o biocerámicas reabsorbibles. En este trabajo, se propone una aproximación, mediante diferentes técnicas de microscopía, espectroscopía y difracción de rayos X, a la variabilidad histológica y mineral del tejido óseo en el transcurso de la ontogenia y así, poder realizar una extrapolación que permita comprender mejor el implante de materiales biodegradables. Respecto a la variabilidad histológica, se han observado diferentes tipos de tejidos en la cortical del hueso en las distintas edades, siendo el hueso fibrolaminar principal en etapas tempranas de la vida, y el hueso secundario o haversiano en etapas adultas. Los análisis de espectroscopía Raman e infrarroja indican un aumento en la cristalinidad de la parte inorgánica del hueso a lo largo del ciclo vital. Por último, el estudio del tejido óseo mediante difracción de rayos X muestra la presencia de $\beta$-fosfato de calcio e hidroxiapatito como componentes minerales principales del hueso.
\end{abstract}

\section{Palabras Clave: biomaterial, hueso, hidroxiapatita, Raman, IR, Difracción de rayos X}

\section{Contributions of human paleohistology to the study of biomaterials}

The deep study of archaeological human bone could provide relevant information to biomaterials science, as it could tell how the implant process of bioglasses and bioresorbable ceramics is. In this paper, we propose to study, by means of different microscopic, spectroscopic, and X-ray diffraction techniques, the histological and mineral bone variability throughout ontogeny. Extrapolating this data, we could have a better knowledge of biodegradable materials implant. In different ages, we could notice different tissues in cortical bone: fibrolamellar bone is characteristic of early stages of life while secondary or harvesian bone is in adult individuals. Raman and infrared spectroscopy suggest an increase of critallinity in the inorganic matrix during live. Finally, the X-ray diffraction study of bone tissue shows $\beta$-calcium phosphate and hydroxyapatite as the main mineral bone components.

Keywords: biomaterial, bone, hydroxyapatite, Raman, IR, X-ray diffraction.

\section{INTRODUCCIÓN}

Desde hace algún tiempo se estudian las posibles aplicaciones de determinados materiales en campos como la medicina o la cirugía para su uso en implantes. Un biomaterial puede definirse como aquella sustancia diseñada con una forma específica que, en solitario o como parte de un sistema complejo, puede dirigir un proceso diagnóstico o terapéutico en medicina o veterinaria controlando las interacciones entre el material y el organismo vivo (1). Las biocerámicas y los vidrios bioactivos son, actualmente, los implantes sintéticos más utilizados (2). Generalmente, se entiende por biocerámicas aquellos materiales cerámicos que, al ser utilizados en la reparación de partes corporales dañadas, pueden lograr un comportamiento fisiológico específico (3). Existen numerosas clasificaciones de estos biomateriales, siendo una de las más comunes aquella basada en el tipo de respuesta del tejido en el que se implanten. Así, se distingue entre bioinertes, bioactivas y reabsorbibles o biodegradables (4). En el primero de los casos no se crea una interfaz entre el hueso y el implante (5), como sucede en aquellas biocerámicas compuestas de alúmina $\left(\alpha-\mathrm{Al}_{2} \mathrm{O}_{3}\right)$ o circona $\left(\mathrm{ZrO}_{2}\right)(4)$. Las cerámicas bioactivas, por el contrario, crean superficies favorables para la adhesión y crecimiento de hueso, destacando en este grupo el uso del hidroxiapatito $\left(\mathrm{HA}, \mathrm{Ca}_{10}\left(\mathrm{PO}_{4}\right)_{6}(\mathrm{OH})_{2}\right)$ (4). Por último, las cerámicas reabsorbibles o biodegradables, como es el caso del fosfato tricálcico (TCP, $\left.\mathrm{Ca}_{3}\left(\mathrm{PO}_{4}\right)_{2}\right)$, se disuelven con el paso del tiempo al producirse un reemplazo gradual del material por los tejidos naturales (4). Estas últimas requieren, por tanto, que el material presente ciertas características tales como una tasa de degradación proporcional a la de regeneración del tejido, o un tamaño de poro apropiado que promueva la invasión celular, para que la unión entre el tejido y el implante sea óptima (6). El concepto de bioactividad, tratado anteriormente, 
tiene su origen sin embargo en el Bioglass ${ }^{\circledR}$, el primer vidrio bioactivo desarrollado a partir de los experimentos de L. Hench en 1971, en el que se descubrió que ciertos vidrios podían formar una capa biológicamente activa en su superficie (2). Desde entonces, los vidrios bioactivos (biovidrios) y las vitrocerámicas bioactivas, al igual que las biocerámicas, son una fuente importante de biomateriales con aplicaciones clínicas.

Conocer en profundidad la estructura del hueso humano es pues un requisito fundamental en el campo de la investigación de materiales biocompatibles, sobre todo en aquellos con posibilidad de ser utilizados en intervenciones quirúrgicas que palien problemas asociados al desarrollo de los huesos (ej. lesiones traumáticas como fracturas de huesos o enfermedades degenerativas como la osteoporosis). Pero, a pesar de ser crítica la investigación sobre la estructura y función del hueso, aun hoy en día existen ciertos aspectos desconocidos en este sentido. El hueso es un material bioproducido y su estudio se puede abordar desde numerosos campos de investigación (ej. Antropología física, medicina, etc.). Por un lado, podemos decir que el hueso es un composite (7), es decir, un biomaterial que presenta una fase orgánica y una fase inorgánica, y que juntas constituyen una estructura altamente jerarquizada (8). La fase orgánica está compuesta por diversas proteínas tales como colágeno tipo I, osteocalcina, osteonectina u osteopontina (9); mientras que la hidroxiapatita $\left(\mathrm{Ca}_{10}\left(\mathrm{PO}_{4}\right)_{6}(\mathrm{OH})_{2}\right)$ y en menor medida el fosfato de calcio $\left(\mathrm{Ca}_{3}\left(\mathrm{PO}_{4}\right)_{2}\right)$, además de otros elementos minoritarios como magnesio $\left(\mathrm{Mg}^{2+}\right)$ o sodio $\left(\mathrm{Na}^{+}\right)$constituyen la fase inorgánica (10). La porción mineral del tejido óseo confiere rigidez a los huesos una propiedad indispensable en el desarrollo de las funciones biomecánicas de los mismos, relacionada con su grado de mineralización o de cristalinidad $(11,12)$. Por otro lado, el hueso es un tejido vivo que no permanece inalterable a lo largo de la vida, sino que experimenta numerosos cambios en tamaño, forma y organización estructural a través de los procesos de crecimiento, modelado (deposición y reabsorción de hueso en superficies complementarias de hueso) y remodelación (reabsorción ósea seguida de deposición en el mismo lugar) (13). Así, la interacción entre las células que componen este tejido y la matriz orgánica e inorgánica del hueso, va variando durante el desarrollo como resultado de los procesos anteriormente nombrados, para dar lugar a distintas tipologías de hueso. Al inicio del crecimiento (durante el desarrollo embrionario) o en periodos de reparación ósea (donde se genera hueso de novo, (7)), el hueso se encuentra constituido fundamentalmente por tejido óseo inmaduro o entretejido (9) (woven bone, también denominado "no laminar") (7)). Este tipo de tejido se deposita muy rápidamente $(>4 \mu \mathrm{m} /$ día), presentando una disposición aleatoria de las fibras de colágeno y los minerales asociados, a ellas $(7,9)$. En la mayoría de los vertebrados, sobre este hueso inmaduro se deposita hueso laminar (lamellar bone), a una tasa mucho menor $(<1 \mu \mathrm{m} /$ día $)$ y con una orientación rigurosa de las fibras de colágeno y sus minerales asociados formando láminas de hueso (7). Sin embargo, en humanos, debido a las características particulares de crecimiento, tras el hueso inmaduro se forma el denominado hueso fibrolaminar (fibrolamellar bone), con una tasa de deposición intermedia entre el hueso inmaduro y el hueso laminar (7). El hueso fibrolaminar atrapa a los vasos sanguíneos inmersos en el hueso entretejido mediante la deposición de láminas concéntricas de hueso dando lugar a unas estructuras conocidas como osteonas primarias
(7). Mediante el proceso de remodelación, las estructuras denominadas BMUs (basic multicelular units), unidades encabezas por osteoclastos encargados de la eliminación del tejido y seguidas por osteoblastos que depositan tejido nuevo (14), reabsorben el hueso fibrolaminar y diferencian hueso secundario o haversiano. Este tipo de hueso presenta unas estructuras características denominadas osteonas secundarias o sistemas de Havers (15) en las que, al igual que en las osteonas primarias, las láminas concéntricas de tejido se organizan centrípetamente alrededor de un canal central (canal de Havers) que contiene vasos sanguíneos y nervios (15). La principal diferencia entre estas osteonas y las encontradas en el tejido óseo fibrolaminar es la presencia de línea de cemento (estructura que se forma como resultado de la reabsorción y deposición de hueso (16)) en las osteonas secundarias (15). Finalmente, el hueso alcanza su configuración adulta. Sin embargo, debemos mencionar que el estudio de la variación histológica del hueso en la ontogenia es complejo, ya que el resultado final de los procesos de crecimiento, modelado y remodelación parece estar fuertemente influenciado por otros factores tales como el sexo del individuo, la actividad física o las diferentes estructuras histológicas y su proporción dentro del mismo hueso (17).

Bajo esta perspectiva, el estudio en profundidad de restos óseos humanos recuperados en contextos arqueológicos puede aportar datos muy interesantes a las líneas de investigación en biomateriales. Con la realización de este trabajo se pretende presentar nuevas perspectivas en dicho campo de estudio, en relación a aquellos materiales utilizados en implantes, mediante una primera aproximación a la variabilidad histológica y mineral del hueso arqueológico a lo largo de la ontogenia. Por un lado, el conocimiento de las estructuras microscópicas del hueso desde el punto de vista de la Biología, así como su cambio a lo largo de las distintas etapas de la vida del individuo puede ayudar a comprender mejor los procesos de modelado y remodelación de este tejido, algo fundamental para poder conocer con mayor profundidad el proceso de implante y acoplamiento estructural de determinados biomateriales. Por otro lado, el análisis de la porción mineralizada del hueso arqueológico, desde la perspectiva del hueso como material bioproducido, nos permitirá conocer su rango de variación referido a composición química, resistencia mecánica, etc., y así aportar datos al campo de estudio de las biocerámicas y los biovidrios para poder encontrar el material más adecuado para su uso en implantes.

\section{MATERIAL Y MÉTODOS}

Como hemos mencionado anteriormente el hueso es un tejido orgánico vivo, de ahí que el estudio de su estructura y composición necesite de planteamientos previos sobre cuál debe ser el abordaje experimental más apropiado. De manera ideal, podríamos decir que el estudio de las dinámicas de crecimiento, modelado y remodelación del hueso debería ser planteado en términos de estudios longitudinales donde se pueda analizar la transformación del tejido óseo en una serie de individuos a lo largo de una parte o de toda su vida. Sin embargo, este tipo de estudios en nuestra especie son obviamente imposibles, ya que analizar el hueso implicaría su parcial destrucción y esto a su vez, un daño innecesario en la persona. Es por esto que, desde el ámbito de la 
Antropología física, este tipo de abordajes suelen realizarse utilizando material de yacimientos arqueológicos, aunque eso conlleve la inferencia de datos con poblaciones del pasado que no tuvieron por qué estar sometidas a las mismas condiciones que las actuales. Aun así, los análisis sobre hueso arqueológico también son semi-destructivos, por lo que solamente deben ser considerados cuando exista un excedente de material y además éste no sea necesario para conocer datos relativos a la población de origen (ej. estructura demográfica de la población o a la frecuencia de aparición de signos relacionados con su tipo de su vida).

En el presente trabajo se utiliza material procedente de una colección osteoarqueológica de la localidad de Almansa (Albacete), datada entre los siglos XIII y XVIII. Los restos óseos, pertenecientes a un osario, fueron encontrados cerca de la antigua iglesia medieval de Santa María de la Soledad por lo que podrían corresponder a restos de enterramientos de este templo. Al ser restos óseos encontrados en un osario de proporciones considerables, tras su identificación antropométrica, se pudo plantear su utilización en este tipo de exploraciones. Concretamente, para la realización de este estudio se seleccionaron cuatro tibias izquierdas correspondientes a cuatro individuos diferentes. Siguiendo las pautas establecidas por Scheuer y Black (18) referidas al grado de fusión de las epífisis, se determinaron tres categorías de edad: infantil, juvenil y adulto.

Para conocer en profundidad la estructura microscópica del hueso se realizó: (a) un análisis de la histología e histomorfometría del hueso y (b) un estudio del contenido inorgánico del hueso. Para ello, fue necesaria la puesta a punto de un protocolo de preparación de láminas delgadas de hueso arqueológico basado en los propuestos por Frost (1958) (19) y Ubelaker (1974) (20). Las muestras se analizaron en microscopía óptica de transmisión, espectroscopía Raman, espectroscopía de infrarrojos con transformada de Fourier (FT-IR) y difracción de rayos X (DRX), por ser algunos de los métodos más empleados para la caracterización química de la zona mineral del hueso (11). Los dos primeros métodos son complementarios y en general, herramientas muy útiles para el examen de este tipo de tejidos (12).

\subsection{Preparación de láminas delgadas en hueso arqueológico.}

Utilizando una cortadora manual, se realizaron dos cortes en la zona media de la diáfisis de cada una de las tibias $( \pm 2 \mathrm{~cm}$ desde el punto medio), obteniendo dos secciones transversales de hueso de $2 \mathrm{~cm}$ cada uno, es decir, ocho fragmentos en total. Cada fragmento se embutió en resina (EpoFix Resin) a la cual se añadió un catalizador (EpoFix Hardener) y se dejó 24 horas para endurecer. Mediante una bomba de vacío se consiguió que la resina se introdujera por todos los poros del material. De cada uno de los fragmentos anteriormente descritos se obtuvieron cortes de $1 \mathrm{~mm}$ de espesor mediante una microcortadora (Buehler ${ }^{\circledR}$. ISOMET ${ }^{\mathrm{TM}}$. Low speed saw. Lake Bluff. Illinois, USA). De cada fragmento se realizaron tres cortes, con lo que finalmente se consiguieron 24 láminas de 1 mm de espesor.

Puesto que estas láminas se observarían posteriormente en microscopía óptica, el espesor máximo de las mismas había de ser menor o igual a $100 \mu \mathrm{m}$. Para conseguirlo se utilizó una pulidora manual (Metaserv ${ }^{\circledR}$ 3000. Variable speed grinder. Buehler ${ }^{\circledR}$ ). Mediante una lija de carburo de silicio de tamaño de grano 4000, se eliminaron las marcas producidas por la microcortadora de una de las caras de los cortes. Dichas superficies pulidas se fijaron a un portaobjetos mediante un adhesivo de montaje para superficies biológicas (DEPEX Mounting Media). Manualmente y mediante el uso de lijas de carburo de silicio de tamaño de grano 320, 1200, 2500 y 4000 sucesivamente, y a una velocidad media de 150 revoluciones por minuto, se consiguió el espesor requerido de $100 \mu \mathrm{m}$ en las láminas de hueso. El tiempo de uso de cada una de las lijas fue variable dependiendo del estado del corte, adecuándolo en cada caso al tipo de lija más conveniente.

De las láminas de $100 \mu \mathrm{m}$ conseguidas se seleccionaron cuatro (una por cada una de las tibias) y se observaron en el microscopio óptico de transmisión (Olympus BX61). Mediante la cámara incorporada al microscopio (Olympus DP70) y un sistema de software de toma de imágenes (DP Controller 1.1.165., 2001-2002 ${ }^{\circ}$, Olympus Optical CO., LTD), se fotografió de manera secuencial a un aumento de $4 x$, las secciones transversales de tibia de cada uno de los individuos. Posteriormente, mediante el editor de imágenes Photoshop (Adobe ${ }^{\circledR}$ Photoshop ${ }^{\circledR}$ CS4) se montaron las imágenes para lograr una única fotografía que representara la sección histológica de la tibia. Las medidas histomorfométricas se realizaron con el programa Image $\mathrm{J}^{\circledR}$.

\subsection{Espectroscopía Raman, espectroscopía de Infrarrojos con transformada de Fourier y difracción de Rayos X.}

Por otro lado, cuatro de las ocho porciones embutidas en resina (una de cada individuo) fueron preparadas mediante una máquina de pulido automático (Phoenix ${ }^{\circledR}$ 4000. Sample preparation sistem. Buehler ${ }^{\circledR}$ ) para su posterior análisis en espectroscopía Raman. En el proceso de pulido se utilizó primero un plato de diamante embutido de tamaño de partícula $20 \mu \mathrm{m}$ durante $15 \mathrm{~min}$ a una velocidad de $100 \mathrm{RPM}$ y, después, lijas de carburo de silicio de tamaño de grano 2500 y 4000 durante 10 minutos cada una a la misma velocidad que en el caso anterior. Las muestras obtenidas se estudiaron en el microscopio Raman confocal $\left(\right.$ WITec $\left.^{\circledR}\right)$ iluminado con láser de 514,5 $\mathrm{nm}$ y los resultados obtenidos se procesaron con el programa WITec Proyect 2.02 y Microsoft Excel 2007 (Microsoft ${ }^{\circledR}$ Office).

En tres de las tibias estudiadas (un individuo adulto, un individuo juvenil y un individuo infantil) se realizó, mediante una cortadora manual, un corte de $0,5 \mathrm{~cm}$ de anchura. Esta porción transversal se dividió en 3 partes, por lo que para cada individuo se obtuvo una porción anterior, una medial y una lateral. La mitad de cada uno de estos fragmentos se trató en la pulidora manual (Metaserv ${ }^{\circledR}$ 3000. Variable speed grinder. Buehler ${ }^{\circledR}$ ) utilizando una lija de carburo de silicio de tamaño de grano 4000 para conseguir que tanto la zona perióstica como endóstica del hueso fueran lo más lisas posibles. Posteriormente, dichas muestras se analizaron mediante FT-IR (FTIR Perkin Elmer Spectrum 100. Perkin Elmer ${ }^{\circledR}$ ) y los resultados obtenidos se trataron con el programa informático Microsoft Excel.

La otra mitad de los fragmentos que no fue utilizada para el análisis de espectroscopía infrarroja se trituró mediante un molino vibratorio con recipientes de carburo de wolframio para su posterior análisis mediante DRX en polvo (D8 Advance. Bruker $\left.{ }^{\circledR}\right)$. Los resultados obtenidos se procesaron mediante el programa informático EVA $\left(\right.$ Bruker $\left.^{\circledR}\right)$. 


\section{RESULTADOS}

\subsection{Paleohistología}

Macroscópicamente, el estudio de secciones de hueso nos permite analizar su forma y su compartimentalización en las distintas fases del crecimiento. Por un lado, la Figura 1 muestra un aumento tanto en el área de la zona cortical como en el espacio medular del hueso en el transcurso de la ontogenia. Analizando los datos histomorfométricos, se observa que el hueso infantil presenta un área cortical $\left(95,51 \mathrm{~mm}^{2}\right)$ menor que el hueso juvenil $\left(182,16 \mathrm{~mm}^{2}\right)$, y éste a su vez un área menor que ambos adultos (351,96 $\mathrm{mm}^{2}$ y $371,41 \mathrm{~mm}^{2}$, respectivamente). Lo mismo sucede con respecto al área del espacio medular del hueso, siendo menor en la muestra infantil $\left(54,25 \mathrm{~mm}^{2}\right)$ que en la muestra juvenil $\left(81,60 \mathrm{~mm}^{2}\right)$ y con valores máximos en el Adulto 1 y el Adulto 2 (105,11 $\mathrm{mm}^{2}$ y 131,26 $\mathrm{mm}^{2}$, respectivamente). Además, pueden verse diferencias en cuanto a la contribución de cada una de estas zonas al total del corte, observándose un aumento del área ocupada por la cortical con respecto a la ocupada por el espacio medular con la edad (Fig. 1). Así, en el individuo Infantil la cortical supone un $64 \%$ del total y el espacio medular un 36\% del mismo, mientras que para el hueso juvenil la cortical ocupa un $69 \%$ y el espacio medular un $31 \%$. En los individuos adultos la zona cortical representa aproximadamente las tres cuartas partes del corte ( $77 \%$ en el Adulto 1 y $74 \%$ en el Adulto 2) mientras que el espacio medular supone sólo un cuarto del total ( $23 \%$ en el Adulto 1 y $26 \%$ en el Adulto 2 ).

Microscópicamente, el estudio de hueso arqueológico permite observar las distintas estructuras presentes en el tejido óseo y por lo tanto, analizar la distribución de las distintas tipologías de hueso a lo largo del desarrollo (Fig. 2). Como se observa en las fotografías obtenidas en el microscopio óptico de transmisión, el individuo Infantil (Fig. 2A-B) presenta en

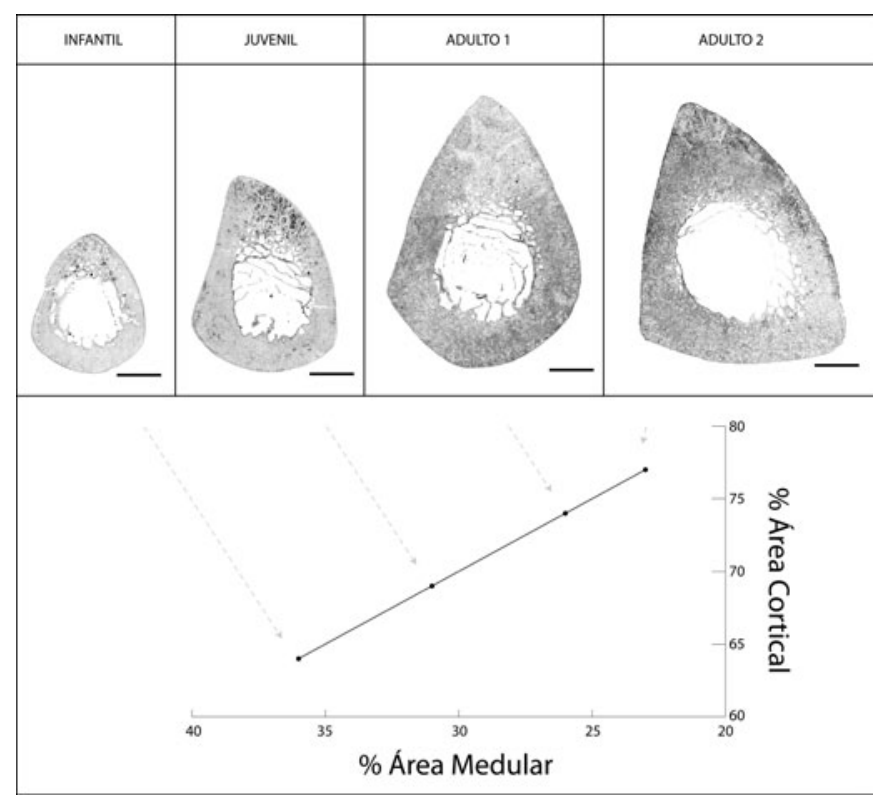

Figura 1. Cortes histológicos de las tibias izquierdas de la población de Almansa. En la parte superior de la imagen se observan los cortes correspondientes a cada una de las etapas del ciclo vital. En la parte inferior se muestra una representación gráfica de la variación en la contribución del área medular y cortical al total del corte en el transcurso de la ontogenia. Las barras de escala representan $5 \mathrm{~mm}$. su cortical tejido de tipo fibrolaminar (HFL, Fig. 2A). La parte media de la cortical aparece ocupada por hueso haversiano (HH, Fig. 2A) en el que se pueden apreciar osteonas secundarias (OS, Fig. 2B). En la parte de la cortical ocupada por tejido fibrolaminar se observan tanto osteonas primarias (OP, Fig. 2B) como osteonas de deriva (drifting osteons) (21) (DO, Fig. 2B), las cuáles parecen presentar cierta alineación. Igualmente, pueden observarse unas estructuras con bordes irregulares, que aparecen distribuidas en diferentes zonas de la cortical y concentradas especialmente en la parte anterior de la tibia denominadas espacios de reabsorción (ER, Fig. 2B), estructuras relacionadas con los procesos de remodelación. El individuo Juvenil (Fig. 2C-D) presenta un aspecto distinto al descrito para el individuo anterior, si bien se pueden observar algunas similitudes. La parte más externa de la cortical presenta, igual que en el caso anterior, hueso fibrolaminar, pero en este caso aparece restringido fundamentalmente a la cortical externa (HFL, Fig. 2C). En este tipo de tejido pueden observarse osteonas primarias (OP, Fig. 2D). El resto de la cortical está ocupado por hueso haversiano (HH, Fig. 2C) compuesto por osteonas secundarias (OS, Fig. 2D). En la parte anterior, al igual que en el caso anterior, se observan, aunque en menor número, espacios de reabsorción. Por último, ambos adultos tienen una estructura microscópica muy similar (Figuras 2E-F), con la mayor parte de la cortical ocupada por osteonas secundarias (OS, Fig. 2E) entre las cuales se observan restos de tejido fibrolaminar constituyendo las denominadas laminillas intersticiales (LI, Fig. 2F). Los espacios de reabsorción han disminuido con respecto a los casos anteriormente descritos y aparecen como algo aislado.

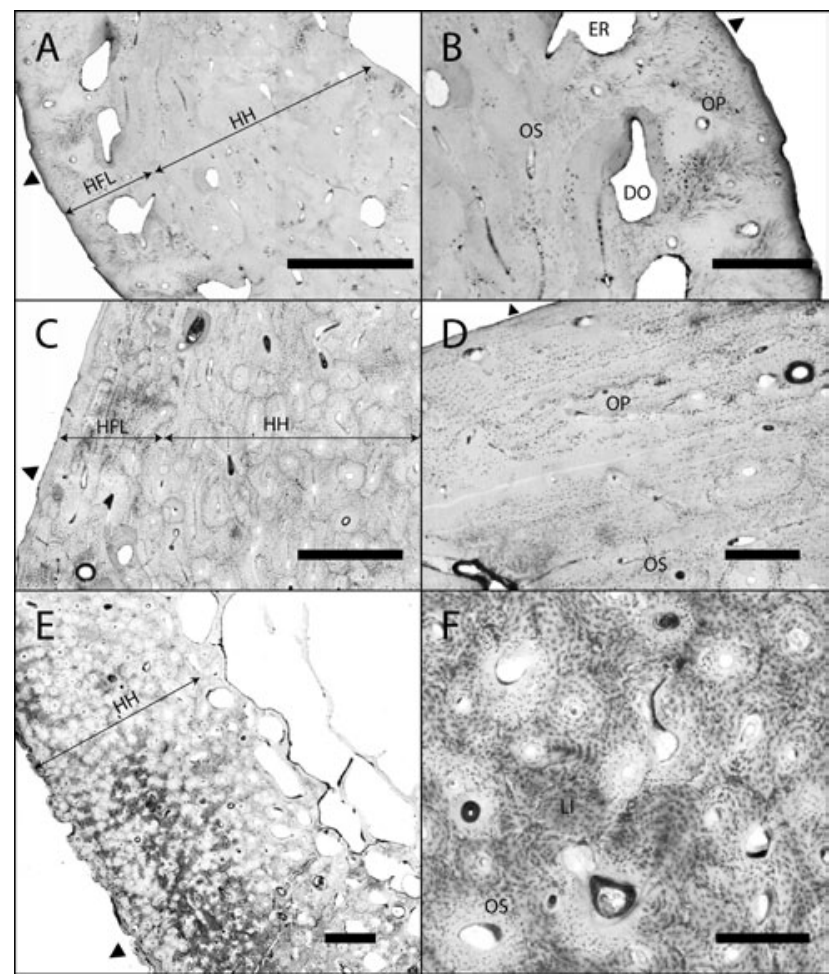

Figura 2. Cortes histológicos en detalle de la cortical de las tibias de los individuos infantil (A, B), juvenil (C, D) y adulto (E, F) (4x). DO = osteona en deriva, $\mathrm{ER}=$ espacio de reabsorción, $\mathrm{HFL}=$ hueso fibrolaminar, $\mathrm{HH}=$ hueso haversiano, $\mathrm{LI}=$ laminillas intersticiales, $\mathrm{OP}=$ osteona primaria, $\mathrm{OS}=$ osteona secundaria. El triángulo señala la parte exterior del corte. Las barras de escala representan $1 \mathrm{~mm}$ para las figuras A, C, E y $400 \mu \mathrm{m}$ para las figuras B, D, F. 


\subsection{Espectroscopía Raman}

La espectroscopía vibracional (Raman, IR) permite caracterizar las diferentes estructuras químicas que aparecen en el tejido óseo. Las diferentes bandas de los espectros se corresponden con modos de vibración de las distintas moléculas que componen el tejido óseo (Fig. 3). En este sentido, los análisis Raman realizados en este estudio, permiten distinguir un pico de intensidad en $960 \mathrm{~cm}^{-1}$ que se corresponde con una banda de $\mathrm{PO}_{4}^{3-}$ perteneciente a la hidroxiapatita (22). De manera general, los análisis de espectroscopía Raman muestran que, en el transcurso de la ontogenia, el hueso presenta diferencias con respecto al grado de cristalinidad de su componente mineral principal: la hidroxiapatita. Esta información se obtiene a partir de la intensidad de fluorescencia de los distintos componentes minerales (representada en el eje $\mathrm{Y}$ de las gráficas), ya que una mayor intensidad en el espectro Raman indica menor organización o cristalinidad. Para comprobar que los resultados del análisis Raman no mostraban información relacionada con factores extrínsecos a la química inorgánica del hueso (p. ej., materia orgánica presente en el hueso), se realizó un análisis térmico diferencial y termogravimétrico (ATD-TG). Los resultados de este análisis complementario indican que la materia orgánica presente en la muestra no interfiere en el resultado obtenido en espectroscopía Raman ya que todos los individuos presentan la misma cantidad de ésta $(\approx 25 \%)$. De manera general, se observa cómo el hueso perteneciente al individuo Infantil es menos cristalino en su estructura mineralógica que el hueso del individuo Juvenil, y éste menos ordenado o cristalino a su vez que ambos adultos (Fig. 3).
Pero éstas no son las únicas diferencias observadas. En lo relativo al individuo Infantil (Fig. 3), se observan diferencias entre las diferentes zonas tanto de la cortical como de la tibia. Por un lado, la cortical interna presenta una estructura más amorfa (menos cristalina) en la zona anterior y lateral de la tibia, mientras que en las zonas medial y posterior, es la parte media de la cortical la que presenta menor organización. Para el individuo Juvenil (Fig. 3) aparece una semejanza en cuanto a estructura amorfa de la cortical dos a dos, como para el caso anterior. Sin embargo, tanto las zonas de la tibia como las partes de la cortical difieren con respecto a las del individuo Infantil. En el individuo Juvenil, la zona externa de la cortical se corresponde con la estructura menos cristalina en la parte anterior y medial de la tibia. En las zonas lateral y posterior es la zona interna de la cortical la que muestra una estructura más amorfa. En la gráfica correspondiente al individuo adulto (Fig. 3), resulta relevante el hecho de que la parte externa de la cortical es muy amorfa en la zona anterior de la tibia, en la espinilla, mientras que en el resto de zonas del hueso, todas las partes de la cortical parecen tener una cristalinidad similar.

\subsection{Espectroscopía de Infrarrojos con transformada de Fourier}

Los análisis de FT-IR (Fig. 4) muestran resultados complementarios a los obtenidos mediante espectroscopía Raman, ofreciendo información de cómo se organiza la estructura química del hueso. De manera general se observa que todos los espectros mantienen similitudes en los picos que los conforman. En este sentido, se identifican las principales especies moleculares que componen el hueso: fosfato $\left(\mathrm{PO}_{4}^{3-}\right)$

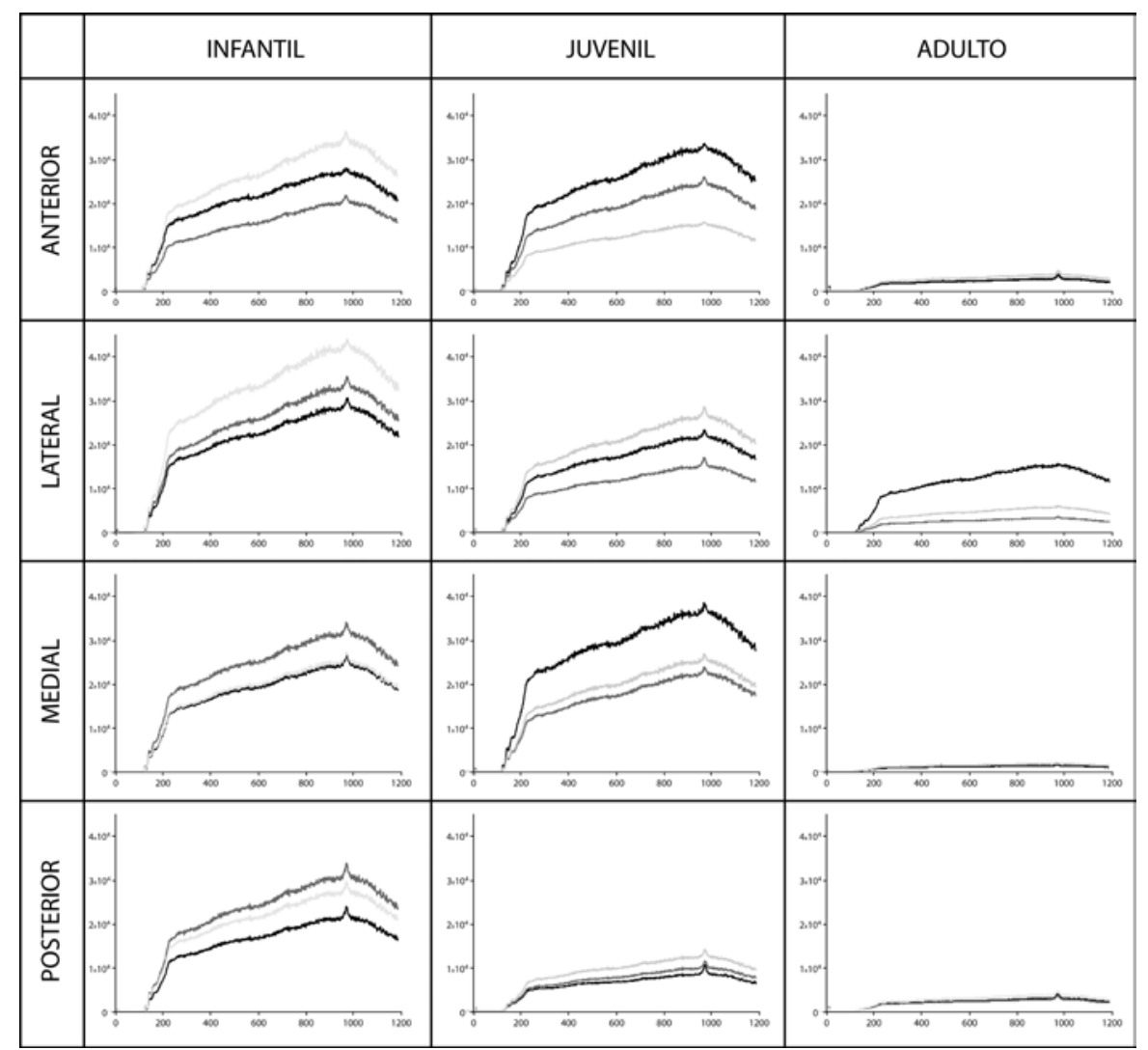

Figura 3.- Espectros Raman de la cortical de las tibias de la colección de Almansa. Se muestran los resultados obtenidos para cada parte de la tibia en cada una de las etapas del ciclo vital. El eje de abcisas representa el desplazamiento Raman $\left(\mathrm{cm}^{-1}\right)$ y el eje de ordenadas la intensidad $(C C D$ cts). Los colores de las gráficas indican las diferentes zonas de la cortical donde se realiza el análisis: negro = cortical externa, gris oscuro = cortical media, gris claro $=$ cortical interna. 
en sus modos de vibración $v_{3}\left(\right.$ en $\left.1033 \mathrm{~cm}^{-1}(23)\right)$ y $v_{1}$ (en 963 $\left.\mathrm{cm}^{-1}(23)\right)$ como componente de la hidroxiapatita, carbonato $\left(\mathrm{CO}_{3}{ }^{2-}\right)$ en su modo de vibración $v_{2}\left(860-890 \mathrm{~cm}^{-1}(23)\right)$ como ión sustitutivo de los grupos hidroxilo y fosfato de la hidroxiapatita y diversas proteínas que constituyen el colágeno tipo I como son la amida I (1630-1700 cm-1 (23)), amida II (1520-1620 $\left.\mathrm{cm}^{-1}(23)\right)$ y amida III (1230-1300 $\mathrm{cm}^{-1}$ (23)) (24) (Fig. 4).

Como característica general, puede observarse una disminución en la intensidad de la señal en el transcurso de la ontogenia (Fig. 4). En el individuo Infantil, no aparecen diferencias entre las intensidades de la señal en la cortical externa e interna, al contrario de lo que se observa en las dos etapas posteriores de juvenil y adulto. En el individuo Juvenil, el espectro correspondiente a la cortical interna tiene una intensidad mayor que la cortical externa, siendo esta relación opuesta para el individuo Adulto.

\subsection{Difracción de Rayos $X$}

El análisis de DRX en polvo complementa, de la misma manera, los datos obtenidos en los análisis anteriores (Fig. 5). El difractograma entre $15^{\circ}$ y $25^{\circ}$ de $2 \theta$ indica que la hidroxiapatita es, de manera general, poco cristalina mostrando una banda muy ancha. Sin embargo, dicha banda es mayor para los individuos Infantil y Juvenil y parece reducirse en el individuo Adulto, en especial en la parte medial de la tibia (Fig. 5).

Además, tras la asignación de diferentes compuestos a estas gráficas, se estima que los componentes principales del hueso son hidroxiapatita y beta-fosfato de calcio $\left(\beta-\mathrm{CaP}_{2} \mathrm{O}_{6}\right)$. La proporción de cada uno de estos componentes al total del hueso, es prácticamente la misma, sin embargo, la parte medial tanto en el individuo Infantil como Adulto parece presentar una mayor presencia de $\beta$-fosfato de calcio que de hidroxiapatita.

\section{DISCUSIÓN}

A pesar de que la variabilidad histológica a lo largo de la ontogenia humana ha sido ampliamente analizada desde múltiples puntos de vista, podemos decir que aún hoy en día existen aspectos que permanecen ciertamente desconocidos. En este sentido, tal y como mencionábamos en la introducción, el presente trabajo realiza una aproximación multidisciplinar al estudio del tejido óseo con la intención de facilitar la interrelación entre las disciplinas científicas de las Ciencias de los Materiales y la Biología. Los datos extraídos de esta aproximación realizada en hueso arqueológico pueden aportar información relevante en el estudio de determinados materiales y su posterior utilización en implantes quirúrgicos. Por un lado, la Biología, y en concreto la Histología, nos ha permitido conocer en detalle el tipo de tejido que se forma en cada etapa del desarrollo. De esta manera, hemos podido describir los cambios relativos tanto al crecimiento del hueso como los referidos a su diferenciación en forma y los relacionados con su remodelado microscópico. Por otro lado, la aproximación al hueso como material nos ha permitido acoplar a esa diferenciación histológica, la variación en la composición química y estructura cristalina a lo largo del desarrollo de un individuo. Hoy en día se sabe que ciertos materiales presentan propiedades osteoconductivas, como por ejemplo las cerámicas biodegradables o los biovidrios, que promueven los procesos de remodelación ósea haciendo que la unión entre el tejido vivo y el material sea lo más perfecta posible (4).

En general, los resultados histológicos nos indican que, a medida que el hueso de un individuo crece, los diferentes tipos de tejidos óseos que lo componen se modifican, tanto en lo que se refiere a su estructura como a su composición. A nivel macroscópico, podemos observar cómo mientras el hueso está en desarrollo, diferentes tipos de tejido óseo se suceden alternándose la distribución de los espacios que albergan tejidos blandos con las zonas mineralizadas. Tal y como señalamos en el apartado de resultados, esta variación relativa a la edad se puede observar a través del estudio de la proporción de hueso cortical frente a espacio medular dentro de una misma sección (Fig. 1). Al igual que otros autores (13), observamos que se produce un aumento con la edad tanto en el área ocupada por la cortical del hueso como en el área ocupada por el espacio medular. En este sentido, se observa que a partir de la etapa juvenil el área ocupada por el espacio medular se va reduciendo como consecuencia de un cambio en la deposición de hueso nuevo que, a partir de este momento, se empieza a depositar en el periostio y endostio del hueso (13). Por otro lado, a nivel microscópico, el primer tejido óseo (hueso entretejido o inmaduro (7)) que aparece en la ontogenia es un hueso formado a una velocidad elevada y su nivel de organización es mínimo. Es por eso que proponemos que este tipo de hueso podría ser el que deberíamos encontrar en las primeras fases del proceso de implante de un biomaterial. En el transcurso de dicho proceso se formarían tejidos similares a los que aparecen durante el desarrollo, es decir, hueso fibrolaminar (Fig. 2) y por último hueso haversiano (Fig. 2). Esto entroncaría con uno de los temas más controvertidos entre la comunidad científica experta en biomateriales, que ya adelantábamos en la introducción. La discusión se centra en cuál sería el tamaño de poro ideal en el material para que sus propiedades osteoconductivas sean

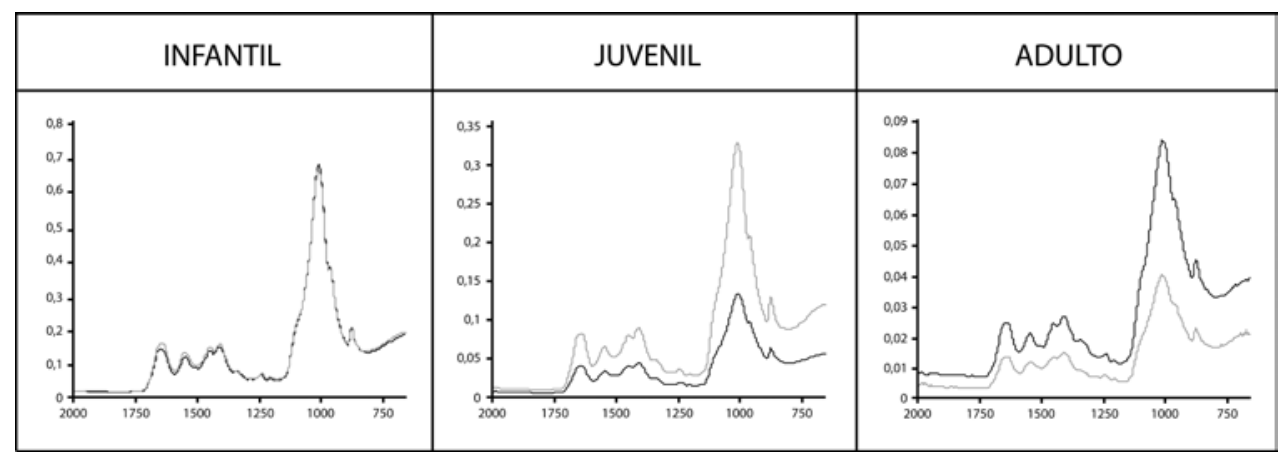

Figura 4.- Espectros de FT-IR de la parte lateral de las tibias de la población de Almansa. En el eje de abscisas se representa la longitud de onda $\left(\mathrm{cm}^{-1}\right)$ y en el eje de ordenadas se representa la absorbancia. Los colores de las gráficas indican las diferentes zonas de la cortical donde se realiza el análisis: negro = cortical externa, gris = cortical interna. 
óptimas (25), ya que la denominada macroporosidad (diámetro de los poros $>100 \mu \mathrm{m}(26))$ permite la migración y proliferación de las células encargadas del proceso de remodelación a las inmediaciones del implante (25). Por lo tanto, el incremento en tamaño y número de poros sería necesario para facilitar el crecimiento del hueso, pero esto repercutiría en las propiedades mecánicas del mismo (25). La variación ontogenética observada en cuanto a las estructuras en las muestras de hueso humano arqueológico (Fig. 2) permite definir como el proceso de remodelación va transformando la cortical del hueso para formar una estructura más compacta, en relación con las propiedades mecánicas del hueso y el estrés fisiológico al que pueda estar sometido (27).

El otro gran bloque de análisis de este trabajo se refiere a la composición química y estructura cristalina del tejido óseo. En primer lugar, es importante destacar que la espectroscopía Raman ha progresado enormemente como técnica para la realización de estudios exploratorios y que su utilización en este estudio ha proyectado resultados muy interesantes (23). En lo que a la edad se refiere, la mayoría de los estudios, así como los resultados obtenidos en este trabajo (Fig. 3), revelan la existencia de cierta tendencia al aumento de cristalinidad con el paso de los años (10) produciéndose un cambio en la cantidad y organización de las especies químicas que forman el hueso (22). Así, los cristales de carbohidroxiapatita van aumentando de tamaño, en longitud y perfección (28) a lo largo de la ontogenia y se van reorganizando haciendo que el hueso sea más rígido y mineralizado (6). En el proceso de reabsorción y posterior deposición ósea que se observa tras el implante de materiales biodegradables o reabsorbibles cabría esperar que el nuevo hueso formado fuera poco cristalino y, con el avance del reemplazo del biomaterial por tejido óseo, fuera aumentando la cristalinidad. Así, la información obtenida en análisis realizados durante el proceso de implante mediante espectroscopía Raman, permitirían, por ejemplo, comprobar la biocompatiblidad entre el hueso y los materiales utilizados en implantes (29). En segundo lugar, tal y como se ha comentado anteriormente, la técnica de FT-IR es complementaria a la espectroscopía Raman, siendo ambas fundamentales en el estudio de tejidos mineralizados
(23). Al igual que con la técnica anterior, el FT-IR nos indica un aumento en la cristalinidad del tejido en el transcurso de la edad (24) (Fig. 4), así como en las distintas zonas de la cortical en distintos momentos de la ontogenia. La relación entre la intensidad transmitida por cada una de las diferentes zonas de la cortical en los diferentes individuos podría estar reflejando el papel de la remodelación en el paso del tiempo, ya que las estructuras histológicas en cada etapa vital son diferentes. Por último, los resultados de DRX concuerdan con los obtenidos por otros autores en lo relativo a la baja cristalinidad de la carbohidroxiapatita presente en el hueso, probablemente debido a la presencia de numerosos iones (30) tales como magnesio $\left(\mathrm{Mg}^{2+}\right)$, carbonatos $\left(\mathrm{CO}_{3}^{2-}\right)$ y fosfatos $\left(\mathrm{HPO}_{4}^{2-}\right)(28)$. La identificación de otro mineral en el hueso arqueológico humano diferente a la carbohidroxiapatita, el $\beta$-fosfato de calcio (Fig. 5), ha sido un resultado extraordinario e inesperado que podría tener cierta relevancia en las perspectivas sobre la búsqueda de los materiales idóneos para implantes reabsorbibles. En este sentido, cabría esperar que el carbohidroxiapatito mineral, por su similitud con el carbohidroxiapatito presente en el hueso (principal componente mineral de este tejido), fuera el material más apropiado para su utilización en biomateriales implantables, pero su baja solubilidad y resistencia mecánica han promovido la búsqueda de otros compuestos más adecuados (3), como por ejemplo, las cerámicas biodegradables. Dentro de este grupo de biomateriales, las más comunes son aquellas compuestas por fosfato tricálcico (TCP) o las formadas por una parte estable de HA y una parte soluble de TCP, conocidas como cerámicas de fosfato de calcio bifásico (BCP) (31). Cuando estos materiales son implantados en el tejido óseo, se produce la disolución parcial de los cristales del compuesto y una precipitación de hidroxiapatita carbonatada similar a la que aparece en el hueso, terminando por transformarse todo el biomaterial en carbohidroxiapatito óseo (26). Sin embargo, la presencia de el $\beta$-fosfato de calcio (Fig. 5) en la estructura mineral del hueso humano arqueológico, podría estar sugiriendo que la transformación del biomaterial a carbohidroxiapatito no tendría por qué ser total, sino que sería posible esperar una conversión parcial, en la que además de este compuesto puedan aparecer otros fosfatos de calcio.

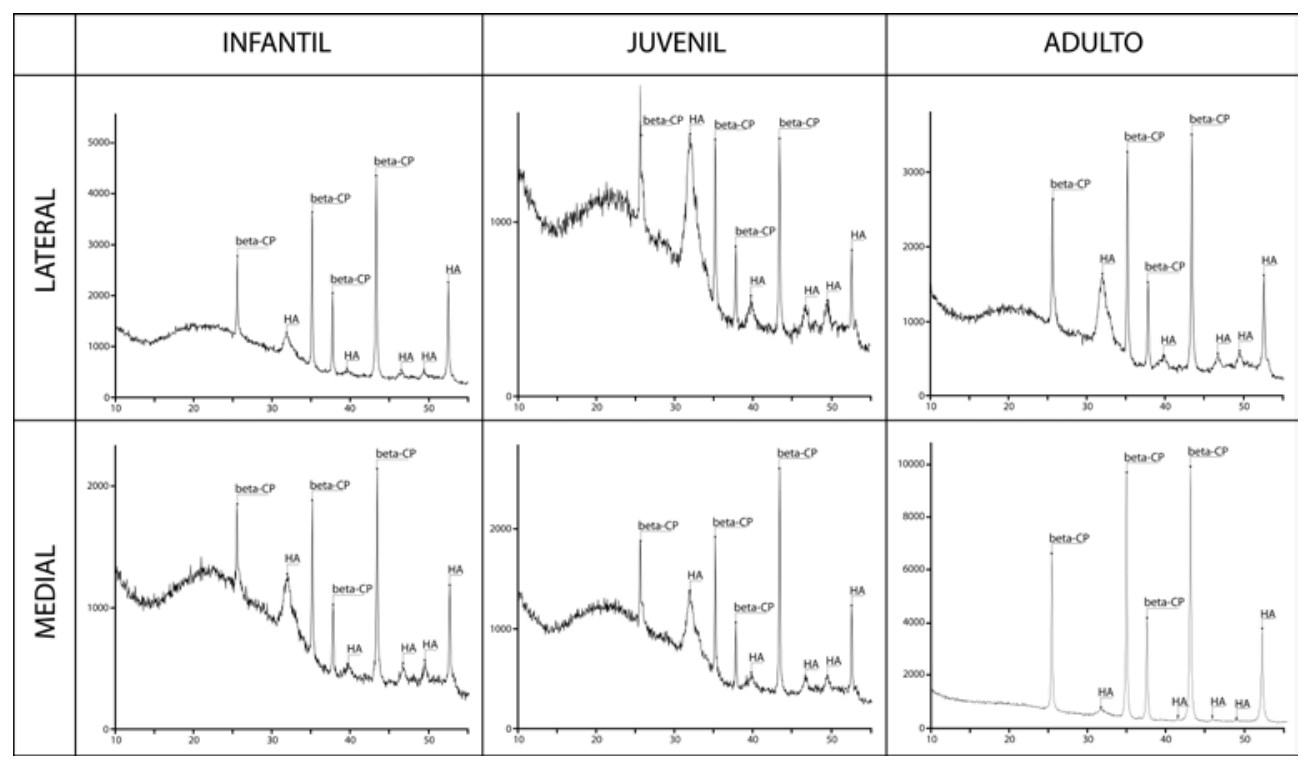

Figura 5.- Patrón de intensidades de las tibias de la población de Almansa para el análisis de difracción de rayos X. En el eje de ordenadas se representa el ángulo $2 \theta$ y en el eje de ordenadas el logaritmo de la intensidad (cuentas). HA = hidroxiapatito; beta- $\mathrm{CP}=$ beta-fosfato de calcio. 


\section{CONCLUSIONES}

- Analizar la relación entre el espacio medular y el tamaño de la cortical es crucial para entender los procesos de crecimiento, modelado y remodelación del tejido óseo. Mediante dichos procesos, tanto la cortical del hueso como el canal medular van aumentando de tamaño, si bien ambas partes del hueso siguen una tasa de cambio diferente, por lo que en diferentes edades la contribución al total de la sección de cada una de ellas es diferente.

- Las diferentes estructuras histológicas que se pueden observar en las distintas etapas del ciclo vital, mediante el estudio de hueso humano arqueológico, pueden servir como plataforma de estudio de los cambios experimentados por las cerámicas biodegradables o los biovidrios, tras su implante en un organismo. Además, el estudio pormenorizado de la porosidad de la cortical del hueso y su cambio en el transcurso de la ontogenia puede ayudar en el debate científico acerca del tamaño de poro ideal de los biomateriales.

- De la misma manera, las diferencias de cristalinidad observadas mediante las distintas técnicas espectroscópicas en el transcurso de la ontogenia pueden reflejar los cambios en cristalinidad que van sufriendo los biomateriales comentados anteriormente. De esta manera, se podría encontrar un gradiente de cristalinidad desde que el biomaterial ha sido implantado hasta que se ha producido la completa reabsorción del mismo.

- Los resultados obtenidos en referencia a la composición mineralógica del hueso arqueológico indican la presencia de otros compuestos de fosfato de calcio, además de la carbohidroxiapatita amorfa característica de este tejido. Por esta razón, la transformación química de los biomateriales reabsorbibles no tendría por qué ser total, pudiendo observarse al final del proceso carbohidroxiapatita y otros fosfatos cálcicos en el tejido óseo.

\section{AGRADECIMIENTOS}

Los autores agradecen al arqueólogo y al departamento de Biología de la Universidad Autónoma de Madrid el permiso para la utilización de la colección, así como a los Doctores Alfonso Blázquez Castro y Adolfo del Campo García por sus recomendaciones. Parte de esta investigación ha sido financiada por los proyectos CGL2009-10766, MICCINN-12HAR20100-23106 y MAT2010-17753 del Ministerio de Ciencia e Innovación (España). Actualmente Ministerio de Economía y Competitividad.

\section{REFERENCIAS BIBLIOGRÁFICAS}

1. D. F. Williams. $<<$ On the nature of biomaterials $>>$. Biomaterials 30, 58975909 (2009)

2. P. N. de Aza, A. H. de Aza, P. Pena \& S. de Aza. $<<$ Bioactive glasses and glass-ceramics $>>$. Bol. Soc. Esp. Ceram. V. 45 (3), 1-11 (2006)

3. P. N. de Aza \& S. de Aza. $<<$ Biocerámicas $>>$ pp. 41-64. En Biomateriales, R. Sastre, S. de Aza \& J. San Román editores. Publicado por Faenza Editrice Iberica s.l. (2004)
4. P.N. De Aza, A.H. De Aza \& S. De Aza. $<<$ Crystalline Bioceramic Materials >>. Bol. Soc. Esp. Ceram. V. 44 (3), 135-145 (2005)

5. J. Chevalier \& L. Gremillard. $<<$ Ceramics for medical applications: A picture for the next 20 years $>>$. J Eur Ceram Soc 29, 1245-1255 (2009)

6. M. Vallet-Regí. <<Biocerámicas: evolución y aplicaciones $>>$. An. Quím. 107 (1), 28-35 (2011)

7. J. D. Currey. $<<$ Bones: structure and mechanics $>>$, Princeton University Press, New Jersey (2002)

8. F. Peters, K. Schwarz \& M. Epple. $<<$ The structure of bone studied with synchrotron X-ray diffraction, X-ray absorption spectroscopy and thermal analysis $>>$. Thermochim Acta 362, 131-138 (2000)

9. U. Welsch. $<<$ Histología $>>$, Medica Panamericana, Madrid (2008)

10. A. Carden \& M. D. Morris. $<<$ Application of vibrational spectroscopy to the study of mineralized tissues (review) $>>$. J Biomed Opt 5(3), 259-268 (2000).

11. G. Penel, G. Leroy, N. Leroy, P. Behin, J. M. Langlois, J. C. Libersa \& P. H. Dupas. $<<$ Spectrometrie RAMAN appliquee aux tissus calcifies et aux biomateriaux phospho-calciques $>>$. Bull Group Int Rech Sci Stomatol Odontol 42, 55-63 (2000)

12. J. R. Caeiro-Rey, S. Dapía-Robleda, E. Vázquez-Fernández \& J. M. GarcíaCastellanos. <<Aplicación de la estructura RAMAN al estudio de la ultraestructura ósea en hueso osteoporótico $\gg>$. Acta Ortopedica Gallega 2(1), 3-6 (2006)

13. H. M. Goldman, S.C. McFarlin, D. M. L. Cooper, C. D. L. Thomas \& J. G. Clement. <<Ontogenetic pattering of cortical bone microstructure and geometry at the human mid-shaft femur $>>$. Anat. Record 292, 48-64 (2009)

14. N. Little, B. Rogers \& M. Flannery. $<<$ Bone formation, remodeling and healing $>>$. Surgery 29(4), 141-145 (2011)

15. M. L. Hillier \& L. S. Bell. $<<$ Differentiating human bone from animal bone: a review of histological methods $>>$. J Forensic Sci 52(2), 249-263 (2007)

16. J. G. Skedros, J. L. Holmes, E. G. Vajda \& R. D. Bloebaum. $<<$ Cement lines of secondary osteons in human bone are not mineral-deficient: new data in a historical perspective $>>$. Anat Rec 268A (1), 781-803 (2005)

17. A. G. Robling \& S. D. Stout. $<<$ Histomorphometry of human cortical bone: applications of age estimation $>>$ pp. 148-182. En Biological anthropology of the human skeleton, M. A. Katzenberg \& S. R. Saunders, editores. Publicado por John Wiley \& Sons (2008)

18. L. Scheuer \& S. Black. <<Developmental juvenile osteology $>>$, Academia, London (2000)

19. H. M. Frost. <<Preparation of thin undecalcified bone sections by rapid manual method $>>$. Biotechnic and Histochemistry 33 (6), 273-277 (1958)

20. D. H. Ubelaker. $<<$ Reconstruction of demographic profiles from ossuary skeletal samples. A case study from the Tidewater Potomac $>>$. Smithsonian Institution Press, Washington (1974)

21. B. N. Epker \& H. M. Frost. $<<$ The direction of transverse drift of actively forming osteons in human rib cortex $>>$. J. Bone Jt. Surg. (Am.) 47, 12111215 (1965)

22. O. Akkus, A. Polyakova-Akkus, F. Adar \& M. B. Schaffler. $<<$ Aging of microstructural compartments in human compact bone $>>$. J Bone Min Res 18 (6), 1012-1019 (2003)

23. M. D. Morris \& W. F. Finney. $<<$ Recent developments in Raman and infrared spectroscopy and imaging of bone tissue $>$. Spectroscopy 18, 155-159 (2004)

24. A. Boskey \& N. P. Camacho. $<<$ FT-IR imaging of native and tissueengineered bone and cartilage $>>$. Biomaterials 28, 2465-2478 (2007)

25. V. Karageorgiou \& D. Kaplan. $<<$ Porosity of 3D biomaterial scaffolds and osteogenesis $>>$. Biomaterials 26, 5474-5491 (2005)

26. R. Z. Legeros, S. Lin, R. Rohanizadeh, D. Mijares \& J. P. Legeros. $<<$ Biphasic calcium phosphate bioceramics: preparation, properties and applications $>>$. J. Mater. Sci. Mater. Med. 14, 201-209 (2003)

27. A. H. W. Chan, C. M. Crowder \& T. L. Rogers. $<<$ Variation in cortical bone histology within the human femur and its impact on estimating age at death $>>$. Am J Phys Anthropol 132, 80-88 (2007)

28. A. Boskey $<<$ Bone mineral crystal size $>>$. Osteoporos Int 24(S5), S16-S21 (2003)

29. P. N. De Aza, F. Guitián, C Santos, S. De Aza, R. Cuscó \& L. Artús. $<<$ Vibrational properties of calcium phosphate compounds. 2. Comparison between hydroxyapatite and $\beta$-tricalcium phosphate $>>$. Chem Mater 9, 916-922 (1997)

30. A. Bigi, G. Cojazzi, S. Panzavolta, A. Ripamonti, N. Roveri, M. Romanello, K. Noris Suarez \& L. Moro $<<$ Chemical and structural characterization of the mineral phase from cortical and trabecular bone $>>$. J Inorg biochem 68, 45-51 (1997)

31. G. Daculsi, O. Laboux, O. Malard \& P. Weiss. $<<$ Current state of the art of biphasic calcium phosphate bioceramics $>>$. J. Mater. Sci. Mater. Med. 14, 195-200 (2003)

Recibido: 29/02/2012

Aceptado: 29/11/2012 